review. J. Bioenerg. Biomembr. 35:533-575.

15. Freichel, M., et al. 1999. Store-operated cation channels in the heart and cells of the cardiovascular system. Cell. Physiol. Biochem. 9:270-283.

16. Hunton, D.L., Zou, L., Pang, Y., and Marchase, R.B. 2004. Adult rat cardiomyocytes exhibit capacitative calcium entry. Am. J. Physiol. Heart Circ. Physiol. 286:H1124-H1132.

17. Hunton, D.L., et al. 2002. Capacitative calcium entry contributes to nuclear factor of activated T-cells nuclear translocation and hypertrophy in cardiomyocytes. J. Biol. Chem. 277:14266-14273.

18. Uehara, A., Yasukochi, M., Imanaga, I., Nishi, M., and Takeshima, H. 2002. Store-operated Ca2+ entry uncoupled with ryanodine receptor and junctional membrane complex in heart muscle cells.
Cell Calcium. 31:89-96.

19. Lewis, R.S. 2001. Calcium signaling mechanisms in T lymphocytes. Annu. Rev. Immunol. 19:497-521.

20. Vega, R.B., et al. 2004. Protein kinases C and D mediate agonist-dependent cardiac hypertrophy through nuclear export of histone deacetylase 5 . Mol. Cell. Biol. 24:8374-8385.

21. McKinsey, T.A., Zhang, C.L., Lu, J., and Olson, E.N. 2000. Signal-dependent nuclear export of a histone deacetylase regulates muscle differentiation. Nature. 408:106-111.

22. Zhang, C.L., et al. 2002. Class II histone deacetylases act as signal-responsive repressors of cardiac hypertrophy. Cell. 110:479-488.

23. Chang, S, et al. 2004. Histone deacetylases 5 and 9 govern responsiveness of the heart to a subset of stress signals and play redundant roles in heart development. Mol. Cell. Biol. 24:8467-8476.

24. Liu, Y., Cseresnyes, Z., Randall, W.R., and Schneider, M.F. 2001. Activity-dependent nuclear translocation and intranuclear distribution of NFATc in adult skeletal muscle fibers. J. Cell Biol. 155:27-39.

25. Liu, Y., Randall, W.R., and Schneider, M.F. 2005. Activity-dependent and -independent nuclear fluxes of HDAC4 mediated by different kinases in adult skeletal muscle. J. Cell Biol. 168:887-897.

26. Parsons, S.A., et al. 2004. Genetic loss of calcineurin blocks mechanical overload-induced skeletal muscle fiber type switching but not hypertrophy. J. Biol. Chem. 279:26192-26200.

27. Bers, D.M. 2002. Cardiac excitation-contraction coupling. Nature. 415:198-205.

\title{
A unique role for Stat5 in recovery from acute anemia
}

\author{
Gregory D. Longmore
}

Departments of Medicine and Cell Biology, Division of Hematology, Washington University, St. Louis, Missouri, USA.

\begin{abstract}
The precise role of erythropoietin receptor-activated (EpoR-activated) Stat 5 in the regulation of erythropoiesis remains unclear. In this issue of the JCI, Menon and colleagues present new experimental data that indicate a distinct role for Stat 5 in the regulation of stress-induced erythropoiesis, such as during acute anemic states (see the related article beginning on page 683). A critical function for Stat5 is to promote cell survival, possibly through transcriptional induction of the antiapoptotic protein Bcl-x. In the present experimental system, erythropoietinStat 5 signals did not induce Bcl-x expression but did induce oncostatin$M$. Moreover, oncostatin- $M$ was found to enhance survival of erythroid progenitors. This work differentiates between steady-state (or homeostatic) erythropoiesis and stress-induced erythropoiesis at the level of EpoR signaling.
\end{abstract}

In adults, red blood cell production by bone marrow progenitors maintains the steady-state level of circulating cells. But during times of "stress," such as acute anemia, the erythropoietic response is predominantly generated by hematopoietic progenitors residing in the spleen, at least in mice. In both circumstances, erythropoietin (Epo) and SCF are the central regulators, albeit that compensatory circulating Epo levels are higher during recovery from acute anemia. The study of developmen-

Nonstandard abbreviations used: BMP, bone morphogenic protein; Epo, erythropoietin; EpoR, Epo receptor; EpoR-H mice, mice expressing a truncated form of the EpoR containing the single Stat5-interacting tyrosine residue Y343; EpoR-HM mice, mice expressing a truncated form of the EpoR that is tyrosine null (Y343F); f, flexed-tail; PY, phosphotyrosine.

Conflict of interest: The author has declared that no conflict of interest exists.

Citation for this article: J. Clin. Invest. 116:626-628 (2006). doi:10.1172/JCI27988. tal hematopoiesis has revealed that fetal erythropoiesis and adult erythropoiesis occur in distinct anatomic sites and with distinct regulatory features $(1,2)$, and it has been suggested that adult splenic erythropoiesis more closely approximates fetal liver erythropoiesis than adult bone marrow erythropoiesis. Do these differences between steady-state erythropoietic regulation and the stress-induced erythropoietic response reflect differences in the erythroid progenitors in adult bone marrow versus spleen, differences in Epo/SCF signaling, the activity of other cytokines, or differences in the tissue microenvironment? In this issue of the JCI, Menon and coworkers provide evidence implicating the Epo receptor-activated (EpoR-activated) Stat5 signal as a unique contributor to the stress-induced erythropoietic response and thereby distinguishing homeostatic and stress-induced erythropoiesis at the level of EpoR signaling (3).

\section{Stat5 activation is required for the stress-induced erythropoietic response}

Following engagement of the EpoR, the preassociated tyrosine kinase Jak2 is activated, which then mediates phosphorylation of 8 conserved tyrosine motifs to generate phosphotyrosine (PY) recognition sites within the cytoplasmic tail of the EpoR (Figure 1). These PY sites serve to recruit both positive and negative regulators that effect erythroid progenitor expansion through both prosurvival and proliferative signals. The most proximal PY site, Y343, serves to recruit and activate Stat5; however, the role of Stat 5 in the regulation of erythropoiesis remains controversial (4-8). Menon et al. (3) used genetically engineered mice, developed in the laboratory of James $\mathrm{N}$. Ihle (St. Jude Children's Research Hospital, Memphis, Tennessee, USA), that express a truncated form of the EpoR containing the single Stat5-interacting tyrosine residue Y343 (EpoR-H mice) or express a similarly truncated EpoR that is tyrosine null (Y343F) (EpoR-HM mice) (8). Since these mutants were knocked in to the EpoR gene locus, this allows for physiologic mutant receptor expression in appropriate tissues and cells, in the absence of endogenous WT receptor expression. These mice were subjected to 3 stress conditions: phenylhydrazine-induced acute hemolytic anemia, acute anemia secondary to 5-fluorouracilinduced reduction in number of maturing erythroid progenitors, and anemia follow- 


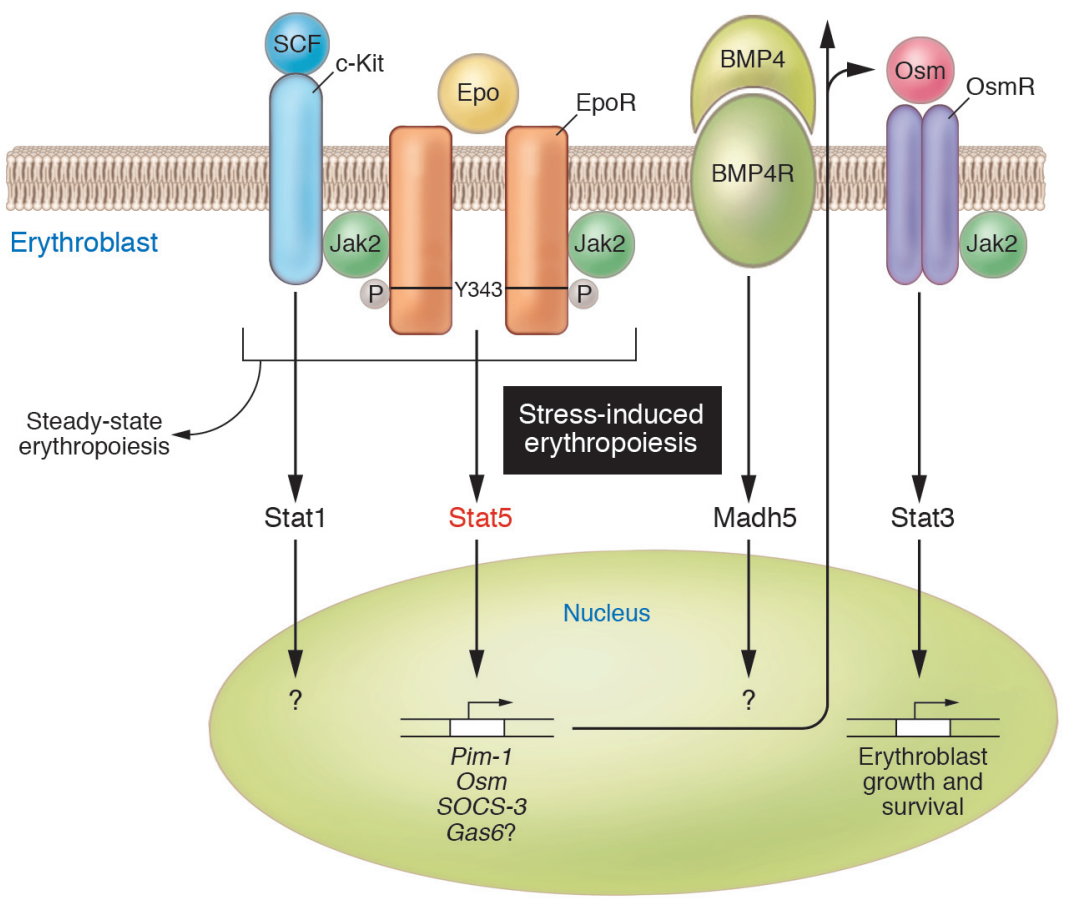

Figure 1

Cytokine regulation of stress-induced erythropoiesis. In this issue of the $\mathrm{JCl}$, Menon et al. report that Stat5, activated by EpoR signals, uniquely regulates stress-induced erythropoiesis (3). Other contributors to stress-induced erythropoiesis include synergy between SCF and Epo, oncostatin-M, BMP4, and possibly Gas6. EpoR-Stat5 signals were found to induce the expression of oncostatin-M, and oncostatin-M cooperates with SCF/Epo signals to enhance erythroid survival. Thus EpoR-Stat5 signals set up a positive feedback loop whereby the signal induces secretion of oncostatin-M, which then contributes to regulation of erythropoiesis during times of acute anemic stress. c-Kit, SCF receptor; Gas6, growth arrest-specific 6; Osm, oncostatin-M; OsmR, oncostatin-M receptor; Pim-1, proviral integration site 1.

ing bone marrow transplantation. In all 3 instances, despite normal, steady-state levels of circulating erythrocytes, EpoRHM mice were incapable of appropriately responding to anemic stress, whereas EpoR-H mice responded in a manner similar to that of WT controls, suggesting that Stat5 is a critical regulator of stressinduced erythropoiesis, but not steadystate erythropoiesis (3). This is supported by the finding that some adult Stat $5 a, b^{-/-}$ mice are also compromised in their response to phenylhydrazine-induced acute anemia (7). A potential limitation of the present study is whether removal of the distal cytoplasmic tail of the EpoR, which has been shown to function as a negative regulatory domain (9), might, in addition to Stat5, also contribute to the stress-induced erythropoietic response.

\section{Stat5-induced oncostatin-M, but not Bcl-x, cooperates with Epo/SCF}

If Stat5 signals are uniquely critical for stress-induced erythropoiesis, then what is the nature of this signal? The authors looked at the induction of a group of known Epo- and Stat5-responsive genes (3). WT EpoR- and EpoR-H-containing primary erythroid progenitors induced expression of Pim-1 kinase, oncostatin-M, and SOCS-3 (Figure 1), but, surprisingly, not $\mathrm{Bcl}-\mathrm{x}$, an antiapoptotic factor important for red blood cell survival (10), whose expression has been reported to be either decreased $(5,7)$ or unaffected in Stat $5 a, b^{-/-}$ mice (6). This result raises the possibility that factors other than, or in addition to, $\mathrm{Bcl}-\mathrm{x}$ are important for the survival of developing red blood cells. Since oncostatin-M has been suggested to contribute to erythroblast survival (11), Menon et al. (3) asked whether oncostatinM signals cooperate with Epo/SCF signals to regulate erythropoiesis. Oncostatin-M treatment significantly enhanced the survival of EpoR-H bone marrow erythroid progenitors in ex vivo cultures, suggesting the presence of a positive feedback loop whereby Epo induces oncostatin-M production and oncostatin-M positively modulates erythroid expansion (Figure 1). How Epo, SCF, and now oncostatin-M signals converge to effect erythroid expansion and whether this is unique to stressinduced erythropoiesis, in vivo, remain to be determined.

\section{Are bone marrow and splenic erythroid progenitors distinct?}

Are EpoR-H signals unique to adult splenic progenitors? In other words, are adult splenic erythroid progenitors distinct from adult bone marrow erythroid progenitors? Apparently not, since ex vivo cultures of EpoR-HM adult bone marrow-derived erythroid progenitors also exhibited defects in Epo-induced proliferation and evidence of increased apoptosis - defects rescued by restoration of the Stat5 PY site (3). But these analyses are, by their nature, performed out of biological context and thus do not account for potential differences in microenvironmental regulation. The recent reexamination of flexed-tail $(f)$ mutant mice is particularly informative in this regard (12). Like Stat5a, $b^{-/-}$and EpoR-HM mice, adult $f / f$ mice exhibit normal steadystate erythropoiesis but do not respond to acute erythropoietic stress. Mutant $f / f$ mice have a mutation in the Madh5 gene, a functional Smad molecule downstream of bone morphogenic protein (BMP) receptor signaling (Figure 1). BMP4 is rapidly, and transiently, induced in the splenic red pulp (site of erythropoiesis) in response to acute anemia and was found to stimulate immature progenitors to give rise to Epo-responsive progenitors. Importantly, only spleen, not bone marrow, progenitors responded to BMP4 in ex vivo cultures. This result suggests that the spleen does indeed contain a unique erythropoietic microenvironment that may distinctly influence erythroid progenitors present therein. Likely, BMP4 expression will be induced in the spleen of both EpoR-HM and EpoR-H mice, since BMP4 transcription appears to be regulated by hypoxia-responsive elements (11). Whether immature, Epo-nonresponsive splenic progenitors from EpoR-HM and EpoR-H mice differ in their response to BMP4 warrants testing, since BMP4 can affect CNS stem cell fate in a pathway activating Stat3 (13).

Finally, studies such as that of Menon et al. (3) highlight the importance of studying and disseminating genetically modi- 
fied mice with minimal or no steady-state phenotype. In many ways these mice could be viewed as models for otherwise normal adult humans who exhibit exaggerated or unexpected responses to inflammation, infectious agents, or cancer progression. As such, they have the potential to identify and dissect regulatory pathways that influence but do not cause disease.

\section{Acknowledgments}

I would like to thank J. Palis for helpful comments.

Address correspondence to: Gregory D. Longmore, Departments of Medicine and Cell Biology, Division of Hematology, Washington University, 660 South Euclid Avenue, St. Louis, Missouri 63110, USA.
Phone: (314) 362-8834; Fax: (314) 362-8826;

E-mail: glongmor@im.wustl.edu.

1. Palis, J., and Segel, G.B. 1998. Developmental biology of erythropoiesis. Blood Rev. 12:106-114.

2. Obinata, M., and Yanai, N. 1999. Cellular and molecular regulation of an erythropoietic inductive microenvironment (EIM). Cell Struct. Funct. 24:171-179.

3. Menon, M.P., et al. 2006. Signals for stress erythropoiesis are integrated via an erythropoietin receptorphosphotyrosine-343-Stat5 axis. J. Clin. Invest. 116:683-694. doi:10.1172/JCI25227.

4. Teglund, S., et al. 1998. Stat5a and Stat5b proteins have essential and nonessential, or redundant, roles in cytokine responses. Cell. 93:841-850.

5. Socolovsky, M., Fallon, A.E., Wang, S., Brugnara, C. and Lodish, H.F. 1999. Fetal anemia and apoptosis of red cell progenitors in Stat5a-/-5b-/- mice: a direct role for Stat5 in Bcl-X(L) induction. Cell. 98:181-191.

6. Marine, J.C., et al. 1999. SOCS3 is essential in the regulation of fetal liver erythropoiesis. Cell. 98:617-627.
7. Socolovsky, M., et al. 2001. Ineffective erythropoiesis in Stat5a(-/-)5b(-/-) mice due to decreased survival of early erythroblasts. Blood. 98:3261-3273.

8. Zang, H., et al. 2001. The distal region and receptor tyrosines of the Epo receptor are non-essential for in vivo erythropoiesis. EMBOJ. 20:3156-3166.

9. D'Andrea, A.D., et al. 1991. The cytoplasmic region of the erythropoietin receptor contains nonoverlapping positive and negative growth-regulatory domains. Mol. Cell. Biol. 11:1980-1987.

10. Wagner, K.U., et al. 2000. Conditional deletion of the $\mathrm{Bcl}-\mathrm{x}$ gene from erythroid cells results in hemolytic anemia and profound splenomegaly. Development. 127:4949-4958

11. Tanaka, M., et al. 2003. Targeted disruption of oncostatin $\mathrm{M}$ receptor results in altered hematopoiesis. Blood. 102:3154-3162.

12. Lenox, L.E., Perry, J.M., and Paulson, R.F. 2005. BMP4 and Madh5 regulate the erythroid response to acute anemia. Blood. 105:2741-2748.

13. Rajan, P., Panchision, D.M., Newell, L.F., and McKay, R.D. 2003. BMPs signal alternately through a SMAD or FRAP-STAT pathway to regulate fate choice in CNS stem cells. J. Cell Biol. 161:911-921.

\title{
An unexpected role for the anaphylatoxin C5a receptor in allergic sensitization
}

\author{
Bart N. Lambrecht
}

Department of Pulmonary Medicine, Erasmus University Medical Center, Rotterdam, The Netherlands.

\begin{abstract}
The anaphylatoxins complement component $3 \mathrm{a}$ and $5 \mathrm{a}$ (C3a and $\mathrm{C5} a$, respectively) are classically seen as proinflammatory mediators of allergic asthma that recruit inflammatory cells, induce edema, and cause bronchoconstriction. A few years ago, controversy arose when it was shown that C5-deficient mice were more susceptible to experimental asthma compared with C5-sufficient mice. In a study by Köhl et al. in this issue of the JCI, it is shown in a series of truly "complementary" experiments that C5a receptor (C5aR) blockade promotes Th2 sensitization upon first exposure to inhaled allergen, whereas C5aR blockade during established inflammation suppresses the cardinal features of asthma (see the related article beginning on page 783). Blockade of C5aR alters the function of airway DCs, crucial for inducing and maintaining Th2 responses in the lung. Targeting C5aR as a treatment for established asthma could be beneficial, but might be accompanied by sensitization to novel antigens.
\end{abstract}

\section{Allergy is mediated by Th2 cells}

The incidence of allergic diseases is currently on the rise. In western societies, up to $25 \%$ of children are sensitized to allergens such as the house dust mite (HDM), pollen, animal dander, or food

Nonstandard abbreviations used: AHR, airway hyperresponsiveness; $\mathrm{C} 3 \mathrm{a}$, complement component $3 \mathrm{a}$; $\mathrm{C} 5 \mathrm{aR}$, C5a receptor; CCL17, CC chemokine ligand 17; CCR4, CC chemokine receptor 4; HDM, house dust mite; mDC, myeloid DC; pDC, plasmacytoid DC.

Conflict of interest: The author has declared that no conflict of interest exists.

Citation for this article: J. Clin. Invest. 116:628-632 (2006). doi:10.1172/JCI27876. components. This sensitization is indicated clinically by the presence in the serum of allergen-specific IgE and by an immediate wheal and flare reaction after skin prick testing with these allergens. als, natural allergen exposure via food or inhalation can lead to allergic diseases such as allergic asthma, allergic rhinitis, or atopic dermatitis. These diseases have an inflammatory component characterized by edema, plasma extravasation, accumulation of eosinophils and mast cells, and overproduction of mucus $(1,2)$. In the case of allergic asthma, an additional symptom In most, but not all, sensitized individu- is airway hyperresponsiveness (AHR) to all kinds of specific and nonspecific stimuli, which is caused by excessive smooth muscle contraction, resulting in airway narrowing. Allergic sensitization is the result of an aberrant Th2 response to allergens. Th2 lymphocytes produce cytokines that control Ig-class switching toward IgE production (e.g., IL-4), allergic eosinophilic inflammation (e.g., IL-5), and AHR (e.g., IL-9, IL-13). In support of a critical role for Th2 cells, experimental asthma does not develop in mice deficient in CD4 cells or most of the above cytokines (3).

\section{The complement system in asthma}

The complement system is crucial for innate host defense because of its formation of a lytic effector system that protects against pathogens. Serine proteases generated in response to classical and alternative activation of complement can cleave the anaphylatoxic peptides complement $3 \mathrm{a}(\mathrm{C} 3 \mathrm{a})$ and $\mathrm{C} 5 \mathrm{a}$ from $\mathrm{C} 3$ and $\mathrm{C} 5$, respectively (4). Various components of the complement pathway have been implicated in mediating allergic inflammation $(5,6)$. First, the anaphylatoxins C3a and C5a are found in increasing concentrations in the bronchoalveolar lavage fluid of asthmat- 